\title{
Pseudomonas aeruginosa and Staphylococcus aureus communication in biofilm infections: insights through network and database construction
}

\author{
Andreia Patrícia Magalhães* (D), Paula Jorge* (D) and Maria Olívia Pereira \\ CEB - Centre of Biological Engineering, LIBRO - Laboratory of Research in Biofilms Rosário Oliveira, University of Minho, \\ Braga, Portugal
}

\begin{abstract}
The polymicrobial nature of most infections is often characterized by complex biofilm communities, where pathogen interactions promote infection progression and severity. Quorum-sensing, the major regulator of virulence and inter-species communication, is a promising target for new anti-infective strategies. This study aimed at collecting and analysing experimental information on the molecular basis of Pseudomonas aeruginosa and Staphylococcus aureus interactions in biofilms. Data were systematically annotated from relevant full-text papers optimally retrieved from PubMed, reconstructed as networks and integrated with specialized databases to identify promising antimicrobial targets. Network analysis revealed key entities regulating $P$. aeruginosa/S. aureus interactions, for instance the PqsABCDE/PqsR quorum-sensing system, which affects $S$. aureus growth and biofilm formation. By identifying the most reported $P$. aeruginosa virulence factors affecting S. aureus, for example, HQNO and siderophores, a list of experimentally validated agents affecting those factors, ranging from synthetic drugs to natural plant extracts, was constructed. The complex experimental data on $P$. aeruginosa/S. aureus interactions were for the first time systematically organized and made publically available in the new Inter-Species CrossTalk Database (www.ceb.uminho.pt/ISCTD).
\end{abstract}

ARTICLE HISTORY

Received 2 May 2019

Revised 28 November 2019

Accepted 29 November 2019

Published online 12 December 2019

\section{KEYWORDS}

Pseudomonas aeruginosa; Staphylococcus aureus; polymicrobial biofilms; quorum-sensing; interspecies communication

\section{Introduction}

Despite important advances in its research, biofilms remain a critical concern for many biomedical applications, being responsible for approximately $80 \%$ of human bacterial infections, such as those associated with the implant of medical devices and chronic infections, for example, lung infections, wounds, among others (Fey 2010; Jorge et al. 2012).

Within the biofilm context, Pseudomonas aeruginosa and Staphylococcus aureus are frequently coexisting pathogens, responsible for co-colonization and coinfection in predisposed patients (Hoffman et al. 2006). The success of these infections is greatly due to their individual pathogenicity emanating from their metabolic versatility (Frimmersdorf et al. 2010), intrinsic and acquired antibiotic resistance (Schmidtchen et al. 2001), biofilm formation (Costerton 2001), and production of multiple virulence factors (Archer et al. 2011; Balasubramanian et al. 2013). Quorum sensing (QS) plays a major role in regulating all these individual traits, but it also controls inter-species communication, phenomenon that often leads to the progression and exacerbation of infection, usually ending in a worse disease prognosis (Castillo-Juárez et al. 2015; Jennings et al. 2017; Sobin et al. 2017).

\section{P. aeruginosa and S. aureus pathogenicity}

P. aeruginosa is a well-known Gram-negative opportunistic human pathogen capable of causing both acute and chronic infections, being highly related to nosocomial infections (Sharma et al. 2014). The lungs are one of the most relevant niches for $P$. aeruginosa colonization, making it one of the most common pathogens isolated from respiratory infections, such as cystic fibrosis (CF) lung infection (Folkesson et al. 2012; RodrigoTroyano and Sibila 2017). Moreover, its great adaptability, phenotypic and genomic plasticity, ubiquity, and opportunistic sense (Shen et al. 2006; Silby et al. 2011; Brown et al. 2012) enables $P$. aeruginosa association with other types of infection, such as burns, wounds (Mulcahy et al. 2014; Turner et al. 2014), and those associated with biomaterials (Breidenstein et al. 2011).

CONTACT Maria Olívia Pereira mopereira@deb.uminho.pt $\Theta$ CEB - Centre of Biological Engineering, University of Minho, Campus de Gualtar, 4710057, Braga, Portugal

*These authors equally contributed to this work.

(C) 2019 Informa UK Limited, trading as Taylor \& Francis Group 
To aggravate this scenario, multidrug-resistant (MDR) $P$. aeruginosa strains are emerging with increasing frequency, rendering ineffective many of the existing antibiotic treatments (Chopra et al. 2008; Boucher et al. 2009). In fact, the World Health Organization (WHO) has recently identified carbapenem-resistant $P$. aeruginosa as a critical threat for which there is an urgent need for new therapies (WHO 2017a). P. aeruginosa displays intrinsic resistance to different types of antibiotics, for example, aminoglycosides, quinolones, and $\beta$-lactams, due to resistance mechanisms such as low outer membrane permeability, multi-drug efflux systems, and inactivating enzymes (e.g. $\beta$-lactamase) (Pang et al. 2019). $P$. aeruginosa can also acquire resistance genes from other microorganisms by horizontal gene transfer, while also being able to experience adaptive resistance, as is the case of biofilm formation (Jorge et al. 2019).

The long persistence of $P$. aeruginosa infections seems to be related with complex mechanisms of adaptation in which virulence factors are expressed according to the infection stage. Several factors have been accounted for the pathogenic potential of this bacterium, with many playing a role in its biofilm formation and dispersal. Such factors include those related with motility (e.g. flagella, pili) (Kazmierczak et al. 2015), enzymes (e.g. proteases) (Lee and Zhang 2015), siderophores (pyoverdine and pyochelin) (Reinhart and Oglesby-Sherrouse 2016), surfactants (e.g. rhamnolipids) (Solano et al. 2014), toxins (e.g. exotoxin A, pyocyanin) (Michalska and Wolf 2015; Hall et al. 2016), and the type-3 secretion system (T3SS) (Hauser 2009).

S. aureus is a Gram-positive human commensal bacteria frequently found in the mucosal surfaces of the nose and respiratory tract, and on the skin (Wendlandt et al. 2013; Lister and Horswill 2014). Consequently, it is easily transmitted by direct contact, making most of the population prone to infection. In the United States of America, a large part of healthy individuals are colonised with S. aureus (30-50\%), within which 1\% are colonized with methicillin-resistant S. aureus (MRSA) (Bhattacharya et al. 2015). Because of this, S. aureus is very often associated with nosocomial infections, since it is commonly transmitted by colonised healthcare staff through direct contact or invasive medical procedures (Bhattacharya et al. 2015). Its ability to evolve and adapt to multiple settings has led MRSA to rapid disperse over the globe, being considered a high threat according to the WHO (WHO 2017b; Jorge et al. 2019). Additionally, MRSA has been developing resistance to virtually all antibiotics classes that are used to treat it, either by making use of its intrinsic resistance factors or by acquiring more through mutations or horizontal gene transfer from other microorganisms (Watkins et al. 2019).

Biofilms of S. aureus are related to many serious acute and chronic infections whose treatment can be complicated since many of its clinical isolates are either MRSA or MDR (Archer et al. 2011; Nair et al. 2014). Furthermore, it has been reported that the presence of S. aureus in heterogeneous biofilms increases the rate of plasmid horizontal transfer, which increases the antibiotic resistance of the biofilm (Venkatesan et al. 2015). The ability of $S$. aureus to survive host immune defences and cause a diverse range of diseases has been attributed to the expression of a broad set of virulence factors (Kong et al. 2016). For example, cell wall-anchored proteins (e.g. clumping factors, fibronectin proteins, protein $A$, and collagen adhesin) enable tissue attachment and evasion of the host immune system, allowing biofilm formation (Foster et al. 2014). Extracellular toxins (e.g. haemolysin, leukotoxin, exfoliative toxin, enterotoxin, and toxic-shock syndrome toxin-1) and enzymes (e.g. coagulase, proteases, and staphylokinase) are secreted to help in tissue penetration and host invasion (Kong et al. 2016). Surface-associated factors are down-regulated and surfactants are expressed in later stages leading to biofilm dispersion and infection spreading (Lister and Horswill 2014). The phenotype of small colony variants (SCV) in S. aureus has also been linked to infection persistence, as they seem to be able to establish intracellular infection and have a reduced metabolic state, thus lowering antibiotic efficacy (Garcia et al. 2013; Proctor et al. 2014). SCV also stimulate a reduced immune response and express increased adhesins and reduced toxins (Tuchscherr et al. 2010).

\section{$\mathrm{P}$. aeruginosa and $\mathrm{S}$. aureus quorum sensing regulation}

QS is a communication mechanism that regulates gene expression in response to fluctuations in cell-population density (Waters and Bassler 2005). In QS, bacteria produce signal molecules, termed auto-inducers (AI), whose concentration increases as a function of cell density (Dixon and Hall 2015). Alterations in gene expression occur when the concentration of an $\mathrm{Al}$ reaches a minimal threshold (Hawver et al. 2016). Usually, Al regulate genes encoding virulence factors, such as those involved in biofilm formation and enhanced motility, but they can also coordinate interactions between microorganisms (intra- and inter-species) and between the microorganism and the host (Knecht et al. 2016; Grandclément et al. 2016). 
Four main QS systems have been identified in P. aeruginosa (Figure 1), namely the Lasl/LasR and the Rhll/RhIR systems (Pesci et al. 1997), the PqsABCDE/PqsR system (Dubern and Diggle 2008), and the AmbBCDE/ lqsR system (Lee et al. 2013). Each system has a corresponding Al: 3-oxododecanoyl-L-homoserine lactone (3-oxo-C12-HSL), N-butanoyl homoserine lactone (C4-HSL), 2-heptyl-3-hydroxy-4-quinolone (Pseudomonas Quinolone Signal - PQS), and 2-(2-hydroxyphenyl)thiazole-4-carbaldehyde (Integrated Quorum Sensing Signal - IQS), respectively (Lee and Zhang 2015). The QS systems regulate the expression of various genes related with motility, biofilm formation, immune evasion, iron scavenging, and antibiotic resistance (Jakobsen et al. 2013), as well as each other, in a hierarchical manner (Figure 1).

The virulence factors expressed by $P$. aeruginosa are diversified and several of them play a role in the process of biofilm development (Pérez-Pérez et al. 2017). For example, the PqsABCDE/PqsR system is responsible for extracellular DNA (eDNA) and lectin production, which are related to biofilm formation and structural stability (Allesen-Holm et al. 2006; Lee and Zhang 2015). In addition, the Rhll/RhIR system controls the expression of rhamnolipids that influence the late stage of biofilm formation and its dispersal (Davey et al. 2003; Lequette and Greenberg 2005). The latter phenomenon is also controlled by the Lasl/LasR system by downregulation the expression of PEL, a major biofilm matrix component (Ueda and Wood 2009). QS also influences swarming motility, which has been linked to early stages of biofilm formation (Shrout et al. 2006) and is also related to the antibiotic tolerance found in $P$. aeruginosa biofilms but not in planktonic cells (Ciofu et al. 2015; Jorge et al. 2019).

As regards to $Q S$ in $S$. aureus, this bacterium deploys a wide collection of virulence factors in order to establish and sustain infection that are primarily coordinated by a complex global regulatory QS system known as accessory gene regulator (Agr). This QS system encodes a signalling circuit that produces and senses the extracellular Al autoinducing peptide (AIP) and the intracellular effector RNAlll (Figure 1) (Recsei et al. 1986; Le and Otto 2015), being responsible for inducing the expression of toxins, which include haemolysins, phenol-soluble modulins (PSM), toxic shock syndrome toxin (TSST), enterotoxins, and Panton-Valentine leukocidin (PVL), among others (Otto 2014; Cheung et al. 2014; Fisher et al. 2018). Apart from toxins, the secretion of several $S$. aureus enzymes, such as proteases, staphylokinase (SAK), and lipases, has been related to the Agr system (Le and Otto 2015; Pietrocola et al. 2017). Biofilm formation has also been strongly associated with Agr function, with downregulation leading to excessive biofilm thickness and lack of structuring, and upregulation inducing biofilm dispersal (Vuong et al. 2000, 2004; Cheung et al. 2011), indicating that the absence of Agr functionality may be advantageous for the success of persistent $S$. aureus infections (Goerke et al. 2000; Otto 2014). Also, the emergence of SCV, strongly associated with chronic and recurrent infections and lack of ability to produce cytolytic toxins, is due to reduced Agr activity and disrupted electron transport chain (Tuchscherr et al. 2011; Pader et al. 2014).

A second QS system, closely related to Agr, is the TRAP/RAP system. The RNAlll-activating peptide (RAP) is an Al that causes phosphorylation of its target protein (TRAP). TRAP is responsible for inducing the production of adhesion proteins, stimulating biofilm formation, and also activates the Agr system. The TRAP/RAP system is mainly active during the early/mid exponential growth phase and it is followed by the Agr system, which is mainly active during the $\mathrm{mid} /$ late exponential growth phase (Ciulla et al. 2019). In addition to these two QS systems, other regulatory systems, namely the staphylococcal accessory regulator (SarA), the S. aureus exoprotein (Sae) operon, and the staphylococcal alternative sigma factor B (SigB), regulate the production of virulence factors and intervene in S. aureus biofilm formation (Zielinska et al. 2012; Le and Otto 2015; Liu et al. 2016).

\section{$\mathrm{P}$. aeruginosa and $\mathrm{S}$. aureus in polymicrobial biofilm infections}

$P$. aeruginosa and S. aureus co-occur in many biofilmrelated infections and the relation between them is known to be competitive in nature (Nair et al. 2014). $P$. aeruginosa can inhibit the growth and even kill $S$. aureus when present in close proximity due to the production of several toxins, such as pyocyanin, hydrogen cyanide, and alkyl-hydroxyquinoline $\mathrm{N}$-oxides, which can block the electron transport chain of $S$. aureus. Accordingly, these toxins also mediate the spatial segregation between the two species (Nair et al. 2014; Stacy et al. 2016). Despite this competition, S. aureus usually persists in $P$. aeruginosa infections, which might be related to the restriction of cell migration in $P$. aeruginosa highly viscous biofilms (Alves et al. 2018). Also, in biofilms where segregation between the two species is not complete, non-resistant $S$. aureus can benefit from being surrounded by resistant $P$. aeruginosa cells (Nair et al. 2014; Stacy et al. 2016). Lastly, S. aureus can employ mechanisms to counteract the effect of $P$. aeruginosa toxins, such as the formation 


\section{P. aeruginosa}

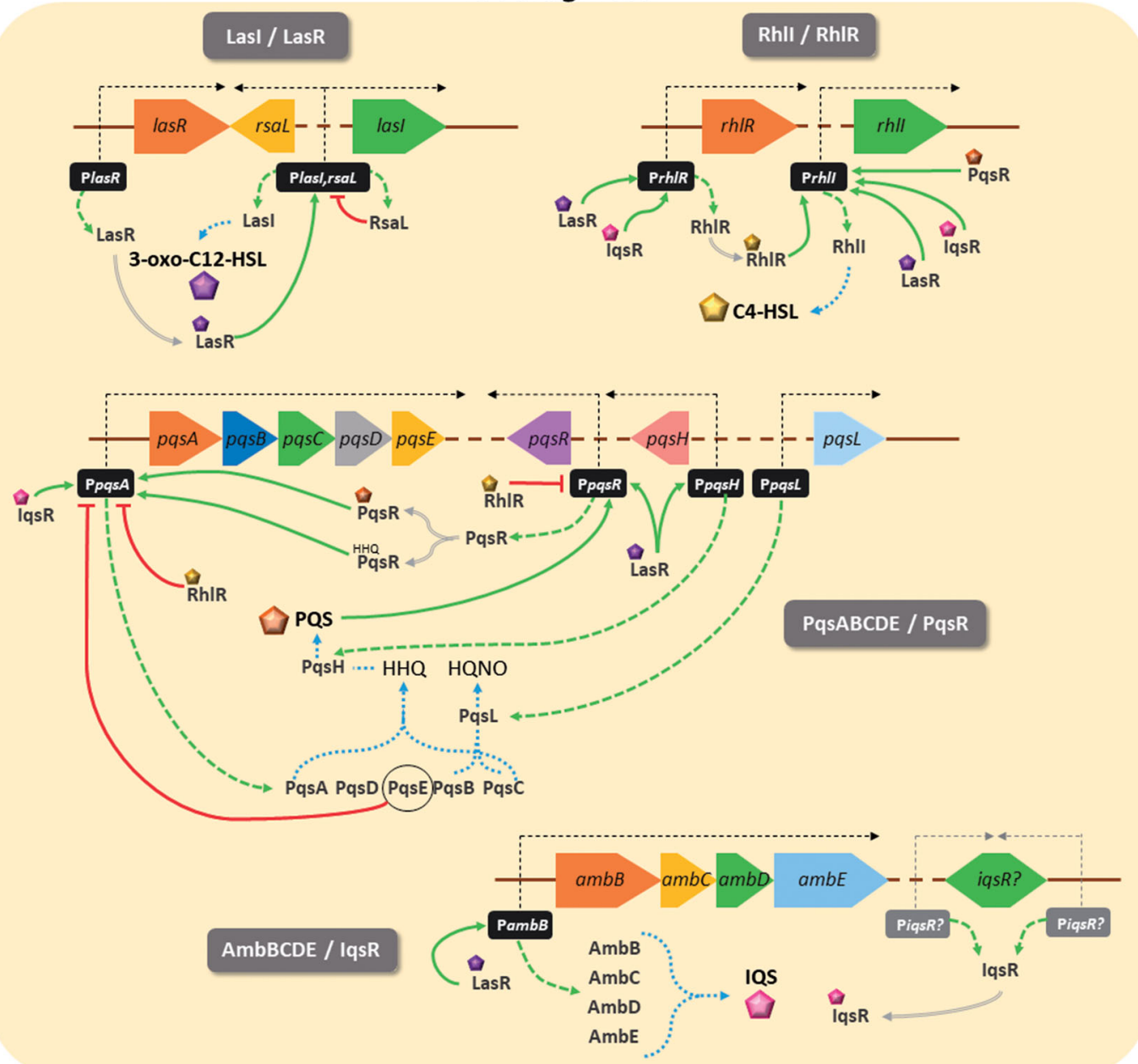

S. aureus

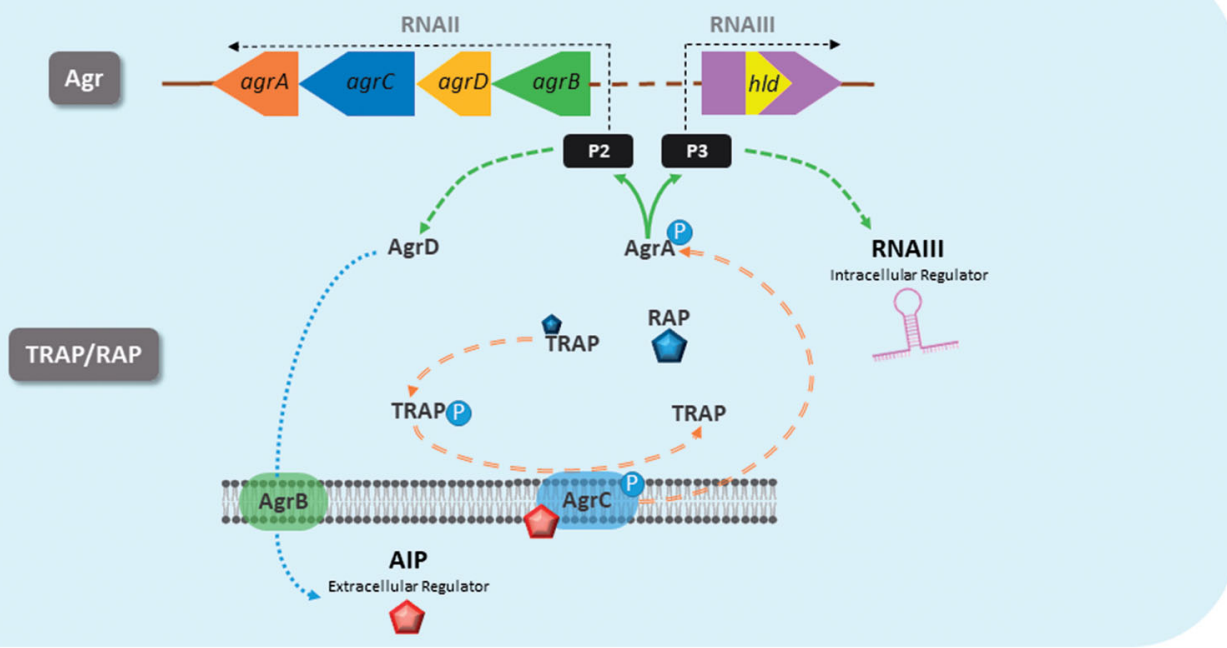

Figure 1. QS regulation in P. aeruginosa and S. aureus. (A) Hierarchical regulation of the four QS systems in $P$. aeruginosa. At the top of the hierarchy is the LasI/LasR system, which, when activated by 3-oxo-C12-HSL, upregulates itself (positive feedback loop) and the other three QS systems. The Rhlrl/RhIR system also upregulates itself and downregulates the PqsABCDE/PqsR system. The latter also has a positive feedback loop and upregulates the Rhlrl/RhIR system. It is noteworthy that this system is responsible for the synthesis of 2-alkyl-4-quinolones, namely PQS, HHQ, and HQNO, which are known to influence $P$. aeruginosa/S. aureus interactions. Finally, the more recently described AmbBCDE/lqsR system upregulates the Rhlrl/RhlR system. (B) S. aureus Agr and TRAP/RAP regulatory systems. The agr operon consists of two transcriptional units, RNAll and RNAlll, driven by the promoters 
of SCV (Nair et al. 2014). All these factors increase the antimicrobial resistance of the polymicrobial biofilm and enhance the severity of the related infection.

Despite new insights into the complexity and impact of multi-species infections on human health, the impact of polymicrobial interactions on infectious diseases remains challenging to examine in laboratory context (Lyczak et al. 2002; Rogers et al. 2010; Price et al. 2013; Filkins et al. 2015). The gap is centred on the dearth of appropriate in vitro models that accurately reproduce the host environments, particularly the availability of required nutrients and the impact of host immune factors.

This manuscript discusses various aspects of the social behaviour of $P$. aeruginosa and $S$. aureus interactions in infectious diseases, according to experimental findings reported in scientific literature. The main goals are to map the mechanisms underlying those interactions, through the collection and curation of scientific textual evidences, in order to gain insight into the implications that $P$. aeruginosa and S. aureus interactions have on the progression and outcome of polymicrobial infections, and analyse this information to pinpoint critical communication channels to be explored for antimicrobial therapy. Therefore, the main contributions of this work are the reconstruction of a knowledge network of up-to-date experimental results on this subject, the deposition of the gathered data in a newly constructed and publicly accessible database (www.ceb.uminho.pt/ISCTD), and the identification of promising therapeutic targets for $P$. aeruginosa/S. aureus co-infections and their respective reported inhibitors.

\section{Materials and methods}

This section describes the steps followed to obtain and curate the information on $P$. aeruginosa and $S$. aureus communication. Moreover, it presents the bioinformatics tools available to explore the gathered data.

\section{Information retrieval and annotation}

The information needed to reconstruct the $P$. aeruginosal $S$. aureus communication network was retrieved from PubMed (Sayers et al. 2019), with emphasis put on the compilation of experimentally validated interactions between the two species. PubMed queries were optimised in terms of number of hits (too low: relevant papers were missed; too high: too many irrelevant papers and impracticable annotation effort) and scope, which was narrowed to experiments mentioning the names of the two species and common terminology denoting simultaneous culturing (e.g. co-cultivation, co-infection, co-culture, polymicrobial, multispecies, mixed-biofilm, etc.). This ensured that PubMed results encompassed almost all the body of work in the field. Subsequently, the relevance of each retrieved document was assessed and relevant information (e.g. textual mentions to organisms, strains, mode of growth, interaction outcome, diseases, and experimental methods) was annotated from the full-text documents. The entity and interaction categories used in expert annotation are described in Tables 1 and 2, respectively.

\section{Information representation and integration}

The network analysis software Cytoscape (Shannon et al. 2003) was used to represent the gathered data in network format. All the curated data was made publicly available in the newly constructed Inter Species CrossTalk Database (ISCTD), the first database solely focussed on microorganism communication, at www. ceb.uminho.pt/ISCTD. All data were transformed into JSON format and the online database constructed using Visual Studio software and HTML, CSS, JavaScript, and jQuery programming languages. Textual evidences were integrated with pertinent regulatory data in the literature for $P$. aeruginosa. Data on anti-QS agents experimentally validated for $P$. aeruginosa was retrieved from the PCQuorum database (Pérez-Pérez et al. 2017) and integrated with the new gathered data.

\section{Results and discussion}

\section{Database and knowledge network overview}

The gathered data stemmed from an annotation effort that resulted in the reconstruction of a network comprehending a total of 670 interactions between

P2 and P3, respectively. RNAll is a four gene operon, agr BDCA, encoding AgrB responsible for processing and exporting AgrD, the AIP precursor. AIP is an exclusively extracellular Al produced in the mid exponential growth phase, whose threshold levels cause AgrC to autophosphorylate, leading to the phosphorylation of AgrA. AgrA activates RNAlll expression, an intracellular effector responsible for increasing the secretion of $S$. aureus toxins and enzymes. In turn, RAP (RNAll-activating peptide) is another S. aureus Al that, in the initial exponential growth phase, induces the phosphorylation of TRAP, its target protein and master regulator of $S$. aureus pathogenesis, activating it. This causes the passage from the planktonic to the biofilm mode of growth and the activation of the Agr system. The two QS systems are therefore connected and are phase-dependent, with AIP indirectly downregulating TRAP phosphorylation. Solid arrows: upregulation of gene expression; dashed arrows: gene expression; dotted arrows: synthesis process; lines with flat ends: downregulation of gene expression; double solid arrows: receptor agonism; double dashed arrows: activation process. 
P. aeruginosa and S. aureus described in 61 different scientific publications (dating from 1993 until October 2019). The annotated information on $P$. aeruginosa/S. aureus interactions was made publicly accessible at www.ceb.uminho.pt/ISCTD, in which users can perform searches and navigate through the annotated data. As

Table 1. Annotated entity categories.

\begin{tabular}{ll}
\hline Entity Categories & \multicolumn{1}{c}{ Examples } \\
\hline Cell* & P. aeruginosa, S. aureus \\
Gene & pvl, pvdE, pchA \\
QS Molecule or AI & 2-heptyl-4-hydroxy quinoline N-oxide (HQNO), \\
& Pseudomonas Quinolone Signal (PQS) \\
Virulence Mechanism & Biofilm, SCV \\
Virulence Factor & Siderophores, DesB \\
Other & Non-virulent proteins (e.g. TatC), metabolites \\
& (e.g. acetate), cell components \\
& (e.g. peptidoglycan), among others ... \\
\hline
\end{tabular}

*Entity and entity category were termed "cell" when the effector or target was unspecified. illustrated in Figure 2, database users are allowed to do more or less refined searches depending on their specific interests. Specifically, users may search for interactions based on one or two specific entity categories (source and target) or considering a specific interaction direction $(P$. aeruginosa $>S$. aureus or $S$. aureus $>P$. aeruginosa) (Figure $2(\mathrm{~A})$ ). The respective data table is generated, showing an organized view of all interaction details, such as organism strains, mode of growth, experimental methods, observations made by the experts, and PubMed references. Users can then narrow down their search by querying for specific terms within the generated table (Figure 2(B)), for example "biofilm".

Figure 3 depicts a representation of this curated knowledge network, illustrating the complexity and the wide array of entities so far reported as involved in this inter-species interaction. This complexity is also

Table 2. Annotated interaction categories.

\begin{tabular}{ll}
\hline Interaction Category & \multicolumn{1}{c}{ Description } \\
\hline $\begin{array}{l}\text { Upregulation } \\
\text { Downregulation }\end{array}$ & $\begin{array}{l}\text { Increase regarding the expression of a gene/protein. } \\
\text { Decrease regarding the expression of a gene/protein. } \\
\text { Increase in the appearance/amount of virulence mechanisms/factors } \\
\text { (or other molecules) or bacterial growth/viability. } \\
\text { Decrease in the appearance/amount of virulence mechanisms/factors } \\
\text { (or other molecules) or bacterial growth/viability. }\end{array}$ \\
Inhibition & $\begin{array}{l}\text { No alteration in the expression of a gene/protein or in the appearance/amount } \\
\text { of virulence mechanisms/factors (or other molecules) or bacterial growth/viability. } \\
\text { Protective effect of one bacteria onto another. } \\
\text { Aggravation of the co-infection's severity/resilience in comparison with the single-species case. }\end{array}$ \\
Protection &
\end{tabular}

\section{NTER- PECIES CROSS ALK DATABASE}

(A) STEP 1 Choose Interaction Direction

STEP 2 Choose Source Entity Category

STEP 3 Choose Target Entity Category

\begin{tabular}{|l|}
\hline- Please Select -- \\
\hline- Please Select -- \\
*ALL * \\
P. aenuginosa $\rightarrow$ S. aureus \\
S. aureus $\rightarrow$ P. aeruginosa
\end{tabular}

(B)

\begin{tabular}{|l|}
\hline- - Please Select -- \\
\hline - Please Select -- \\
*ALL * \\
Cell \\
\hline Gene \\
\hline Polysaccharide \\
Protein \\
QS Molecule \\
Virulence Factor \\
Virulence Mechanism \\
\hline
\end{tabular}

Virulence Mechanis

ur search!

Search table (such as: type of interaction (eg. downregulation), disease (eg. cystic fibrosis), etc...)

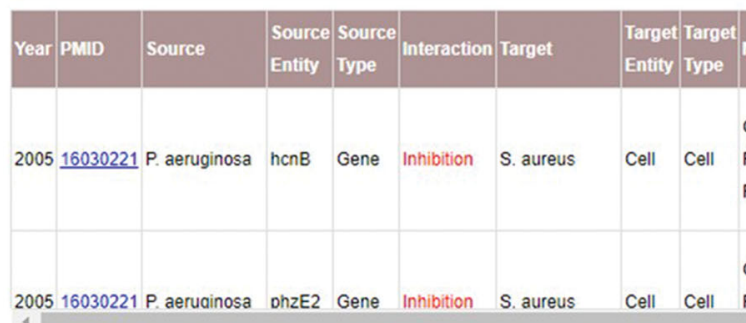

200516030221 P. aeruainosa DhzE2 Gene Inhibition S. aureus

Figure 2. Schematisation of the search flow available in the ISCTD. Users go through (A) a selection flow in which they can specify the interaction direction and the source and target entity categories. When hitting "Search", the database automatically outputs (B) a table containing all annotated data for that search. Users can then search keywords within the table in the search panel. In this figure, the exemplified table shows the effect of $P$. aeruginosa genes on $S$. aureus. 

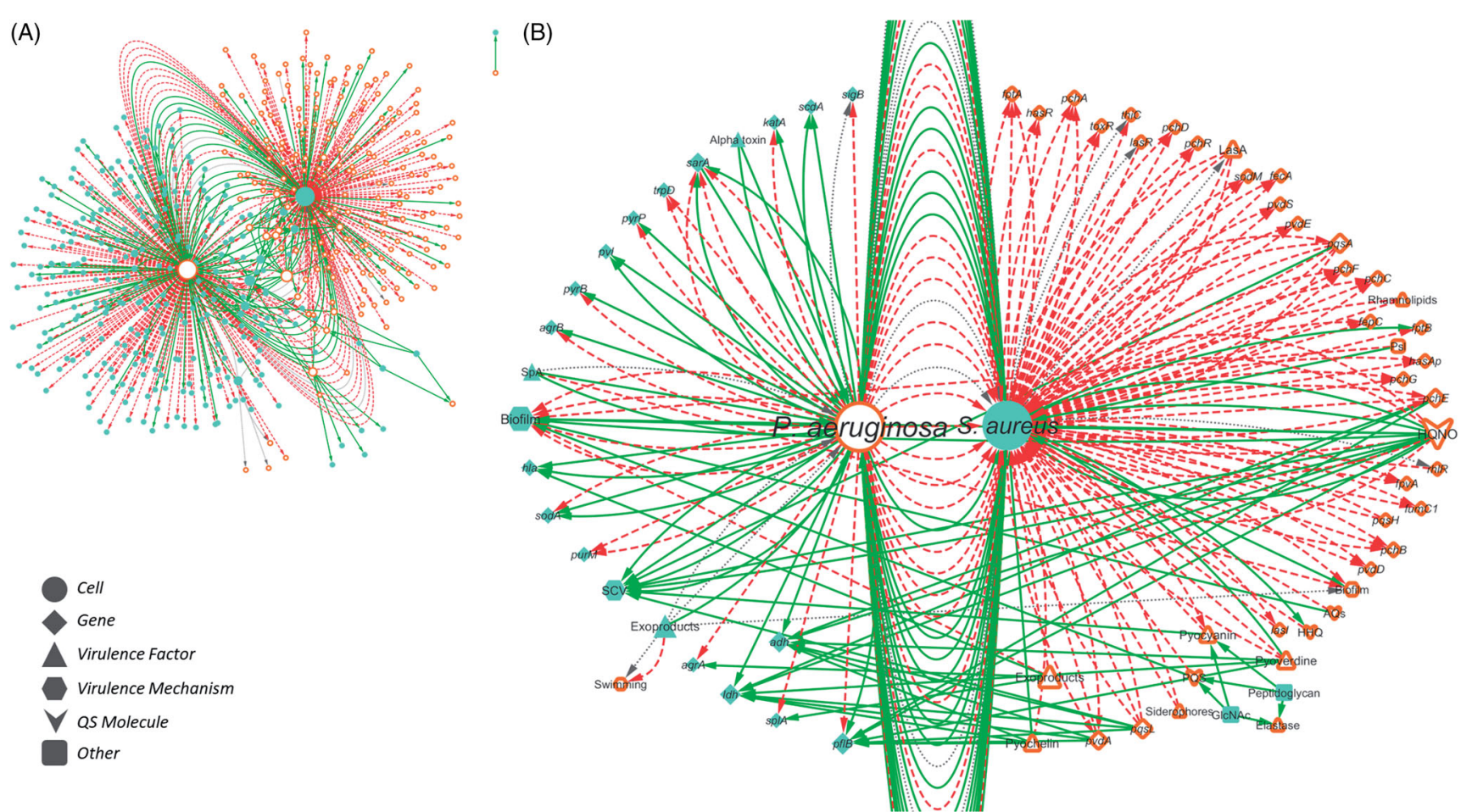

Figure 3. P. aeruginosa/S. aureus interaction network. (A) Overview of all annotated interactions reported in the scientific literature; (B) Most reported interactions (annotated more than once). Solid arrows: positive interactions (e.g. upregulation, stimulation); dashed arrows: negative interactions (e.g. downregulation, inhibition); dotted arrows: null effect. P. aeruginosa and S. aureus nodes are depicted in unfilled and filled shapes, respectively; node and node label sizes are directly proportional to the number of related (outward and inward) edges (interactions).

demonstrated when taking into consideration the different types of interactions, both positive (e.g. stimulation, upregulation) and negative (e.g. inhibition, downregulation) that are established between both bacteria.

All the annotated data is summarised in Table 3 according to interaction directionality, i.e., $P$. aeruginosa $>S$. aureus or $S$. aureus $>P$. aeruginosa interactions, and mode of growth. Interestingly, the majority of the annotated interactions have $P$. aeruginosa as the source microorganism (64\%), which is corroborated by Figure $3(\mathrm{~A})$ in which it is clear that the $S$. aureus node has a greater number of inward edges (interactions) than the $P$. aeruginosa node. In terms of the mode of growth used in all the analysed studies, no major differences were seen between biofilm and planktonic growth, with $46 \%$ of studies performed on biofilms and about $44 \%$ on planktonic bacteria. In vivo was the least studied setting, representing only $11 \%$ of the studies, and was mainly related to clinical studies of human samples rather than in vivo laboratorial testing.

Looking into studies concerning a disease scenario, the majority were CF related (Table 3 ). CF is a genetic disease associated with the dysfunction of a transmembrane chlorine channel that causes secretion of a viscous mucus layer on the respiratory epithelium that facilitates microbial colonisation, with lung disease being the major cause of reduced life expectancy and death in these patients (Scoffone et al. 2019). Although S. aureus is typically one of the primary microorganisms isolated in CF lungs during early childhood, it is quickly followed by $P$. aeruginosa, which progressively increases its prevalence as patients grow older (Razvi et al. 2009; Price et al. 2013). When reaching 18 years of age, the most problematic microorganism for these patients is $P$. aeruginosa, with $80 \%$ being colonised with this bacterium (Høiby 2011), which becomes the most common pathogen isolated from CF sputum at this stage (Folkesson et al. 2012). The problematic of CF lung infection is a great example of the interplay between the two species and may explain why the majority of the studies focus on disclosing the mechanisms through which $P$. aeruginosa and $S$. aureus interact in the CF lung.

Concerning the most reported effector (source) entities, most annotated interactions reported effects of bacteria as a whole (annotated as "cell") (Table 3), meaning that no molecular entity was identified/tested. Most annotated interactions, except those annotated in in vivo studies, have genes as the top target category 
CRITICAL REVIEWS IN MICROBIOLOGY 719

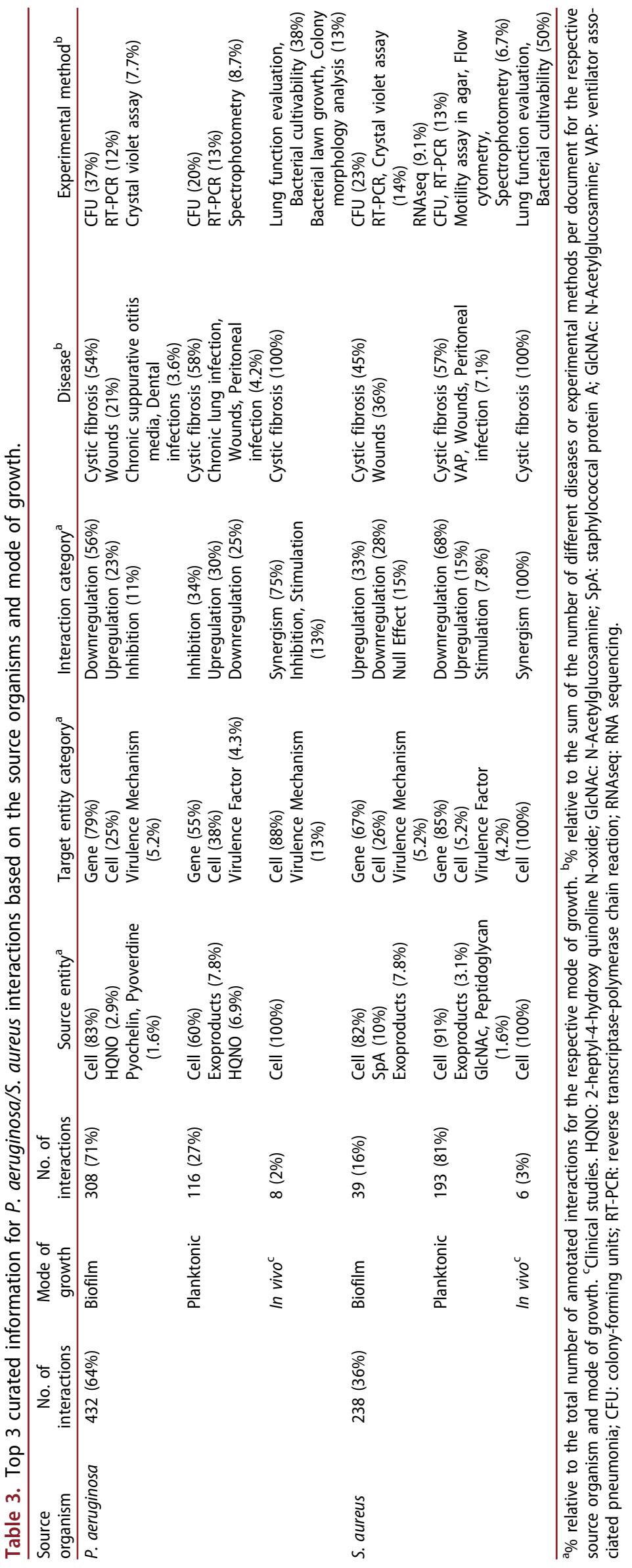


(A)

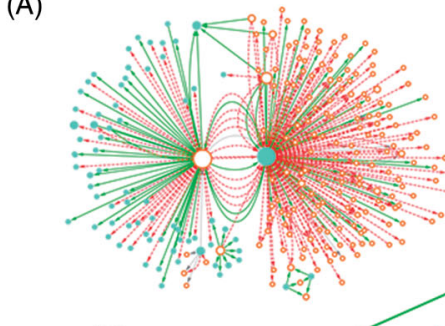

(B)

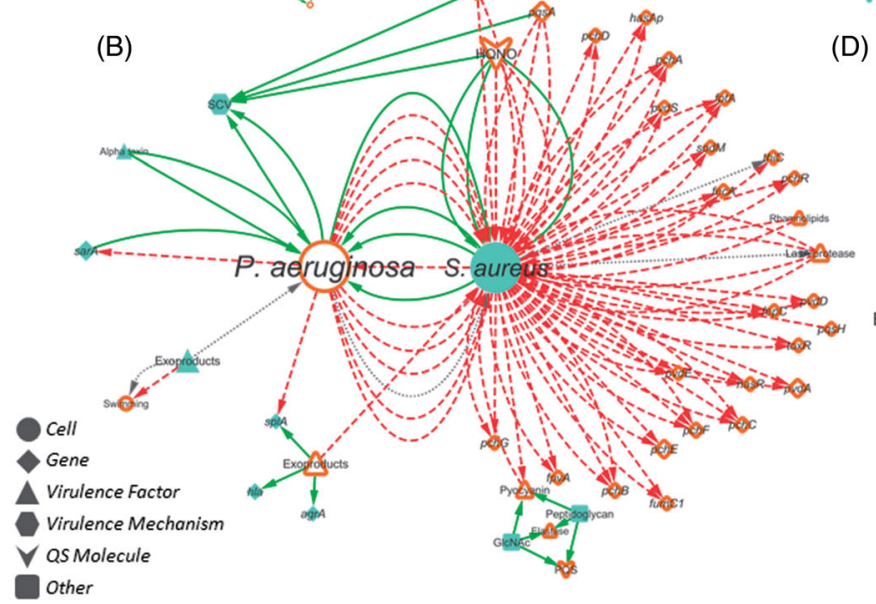

(C)

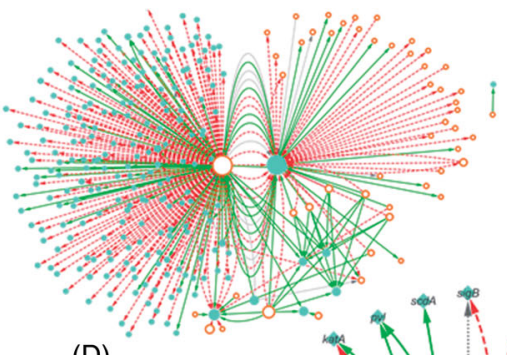

(E)

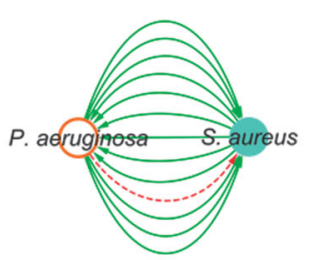

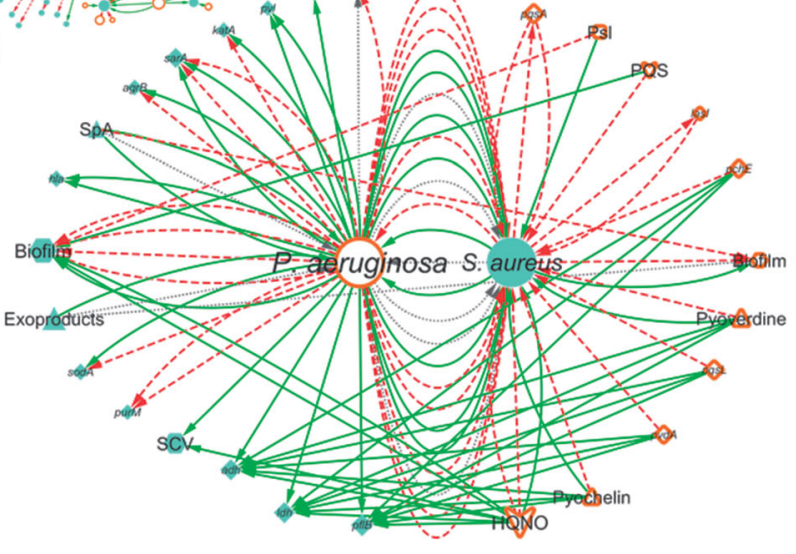

Figure 4. P. aeruginosa/S. aureus interaction networks in planktonic, biofilm, and in vivo settings. (A) All annotated interactions in the planktonic mode of growth; (B) Most reported interactions (annotated more than once) in the planktonic mode of growth; (C) All annotated interactions in the biofilm mode of growth; (D) Most reported interactions (annotated more than once) in the biofilm mode of growth; (E) All annotated interactions in vivo. Solid arrows: positive interactions (e.g. upregulation, stimulation); dashed arrows: negative interactions (e.g. downregulation, inhibition); dotted arrows: null effect. P. aeruginosa and $S$. aureus nodes are depicted in unfilled and filled shapes, respectively; node and node label sizes are directly proportional to the number of related (outward and inward) edges (interactions).

(Table 3). However, this does not translate to a high number of studies analysing gene modulation, which comprise only $11 \%$ of the total analysed, but rather reflects the great amount of results that more in-depth molecular methodologies, such as microarrays and RNA sequencing, output, each translating into an annotated interaction. The concomitant analysis of both bacteria grown as a dual-species biofilm demands the adaptation and optimisation of methodologies typically designed for single-species analysis. This setback can be what is currently impairing a greater use of in-depth methods for the analysis of $P$. aeruginosa and $S$. aureus interactions at the molecular level.

\section{P. aeruginosa and S. aureus biofilm network}

As stated, biofilms are known to be the mode of growth of up to $80 \%$ of human bacterial infections (Fey 2010). Thus, although planktonic testing is practical and informative, studies entailing biofilms do a better job at mimicking a real-life infection scenario. The biofilm, planktonic, and in vivo $P$. aeruginosa/S. aureus interaction networks are represented in Figure 4. Biofilms represent the mode of growth of $52 \%$ of the total annotated interactions, which is still far from ideal.
Interestingly, when looking into $P$. aeruginosa $>S$. aureus interactions, most of them were reported in biofilm scenarios $(71 \%)$, with only $27 \%$ using planktonic growth (Table 3 ). Regarding $S$. aureus $>P$. aeruginosa interactions, planktonic was the most used mode of growth, namely in over $80 \%$ of the annotated interactions. This is also observable in Figure 4, where there is a clear contrast between the number of edges, i.e., interactions, targeting each bacteria when comparing the biofilm (Figure $4(C)$ ) and the planktonic (Figure 4(A)) networks. This observation illustrates a gap in studies concerned with the effect of $S$. aureus on $P$. aeruginosa, revealing an urgent need for more biofilm testing in order to originate a levelled understanding of the bi-directional interplay of both species within mixed consortia.

The most annotated effector entities, apart from "cell", for P. aeruginosa > S. aureus biofilm interactions were HQNO, pyochelin, and pyoverdine (Table 3), as best represented in Figure 4(D). HQNO is a major compound produced by the pqsABCDE operon of $P$. aeruginosa (Figure 1) and is described as antistaphylococcal molecule acting through the inhibition of the oxidative respiration (Williams and Cámara 2009). In turn, pyoverdine and pyochelin are the two major $P$. 
aeruginosa siderophores, that is, iron-chelating molecules, produced in iron-limited conditions (Brandel et al. 2012) that are able to inhibit $S$. aureus biofilm formation. In fact, it has been shown that HQNO and the two siderophores are both required for efficient killing of $S$. aureus by $P$. aeruginosa (Filkins et al. 2015).

In turn, in $S$. aureus $>P$. aeruginosa interactions, the most annotated source entities, apart from "cell", were exoproducts and the staphylococcal protein A (Spa) (Table 3), as best shown in Figure 4(D). Spa is a secreted factor that not only mediates interaction with host cells but has also been shown to bind cell surface structures of $P$. aeruginosa, inhibiting two persistence-associated behaviours of this bacterium: biofilm formation and uptake by host immune cells (Armbruster et al. 2016). The testing of exoproducts was actually generally used in all scenarios and interaction directions, except for in vivo settings. Both $\mathrm{HQNO}$ and staphylococcal protein A are examples of exoproducts, but the general term here annotated was stemmed from studies were a specific molecule was not tested but rather the cell-free supernatant of the bacterial culture. This was an expected feature of these studies given that interspecies communication and QS are mainly based on the production and excretion of molecules to the extracellular media that are sensed by other cells.

As stated, gene was the most annotated target type in all scenarios except "in vivo". For $S$. aureus $>P$. aeruginosa interactions, most annotated target genes in planktonic settings related to the iron binding and transport. Iron is a vital nutrient for bacterial growth but relatively scarce in most infection sites, acting as a limiting factor (Nguyen and Oglesby-Sherrouse 2015). P. aeruginosa secretes exoproducts that negatively impact $S$. aureus growth and survival causing cell death and subsequent iron release, which can be further utilized by P. aeruginosa for its own benefit (Mashburn et al. 2005). The presence of $S$. aureus during co-culture seems to be related with a decreased transcription of $P$. aeruginosa iron regulated genes as $51 \%$ of annotated target genes pertained to this category, with $96 \%$ of them being downregulated, indicating that the presence of $S$. aureus increases usable iron for $P$. aeruginosa. In biofilm settings, QS-related genes, such as $r h I R, p q s H$, and lasl, were one of the top annotated targets (23\%). Looking at the types of established interactions, it is not surprising that $P$. aeruginosa QS genes were downregulated or unchanged in biofilms were $S$. aureus was present. $P$. aeruginosa produces multiple virulence factors that contribute to the removal of $S$. aureus from dual-species biofilms, with the Lasl/LasR, PqsABCDE/PqsR, and Rhlrl/ RhIR QS systems being the major regulators of these factors (Lee and Zhang 2015). In fact, when P. aeruginosa mutants lacking one or both major QS genes (lasl, rhll, and lasl/rhll) were co-cultured in dual-species biofilms with $S$. aureus, a less effective removal of $S$. aureus was observed, suggesting that $S$. aureus thrives when $P$. aeruginosa QS is inactivated (Woods et al. 2018).

As to $P$. aeruginosa $>S$. aureus interactions in planktonic settings, most annotated target gene pertained to functions related to molecule biosynthesis (35\%), mostly of purines and pyrimidines, key components of DNA/RNA synthesis and whose production was upregulated. This could point to an early state of nitrogen starvation in co-culture (Tognon et al. 2019). In biofilm settings, most annotated targets were related with ion and molecule transmembrane transport (17\%) and biosynthesis (13\%), mostly downregulated $(77 \%$ and $93 \%$, respectively), which may contribute to a state of metabolic quiescence, decrease in biofilm formation (Miller et al. 2017), and, possibly, SCV induction.

\section{Discerning $\mathrm{P}$. aeruginosa $>\mathrm{S}$. aureus communication in biofilms}

In clinical settings, biofilm infections caused conjointly by $P$. aeruginosa and $S$. aureus are more virulent than those caused by each independent species, and are frequently associated with increased disease severity and chronicity (DeLeon et al. 2014). Nevertheless, the ecological relationship established between $P$. aeruginosa and $S$. aureus, in such niche, is competitive rather than cooperative. The effect of $P$. aeruginosa in $S$. aureus appears to be preferably studied and will be used here to showcase the complexity and diversity of established interactions.

As demonstrated in Figure 5, a complex and intricate network of regulators dictates the expression of pathogenicity factors in $P$. aeruginosa, which play an important role in its social interplay with $S$. aureus within the dualspecies biofilms. P. aeruginosa secretes several extracellular substances, such as hydroxy-2-alkylquinolines (PQS and $\mathrm{HQNO}$ ) and hydrogen cyanide, which hinder the proliferation of S. aureus biofilms (Palmer et al. 2005; Biswas et al. 2009; Filkins et al. 2015). Both PQS and HQNO are products of the PqsABCDE/PqsR QS system, whose action accounted for 18 of the biofilm annotated interactions in over 5 different documents, including stimulation of $S$. aureus biofilm formation and $S$. aureus growth inhibition (Figure 5). In fact, out of the existing four, this QS system was the most reported as acting, directly or indirectly, upon S. aureus, followed by the Rhll/RhIR system. PQS is a signalling molecule that positively regulates a subset of QS dependent genes involved in siderophore-mediated iron uptake and iron chelating activity in $P$. aeruginosa 


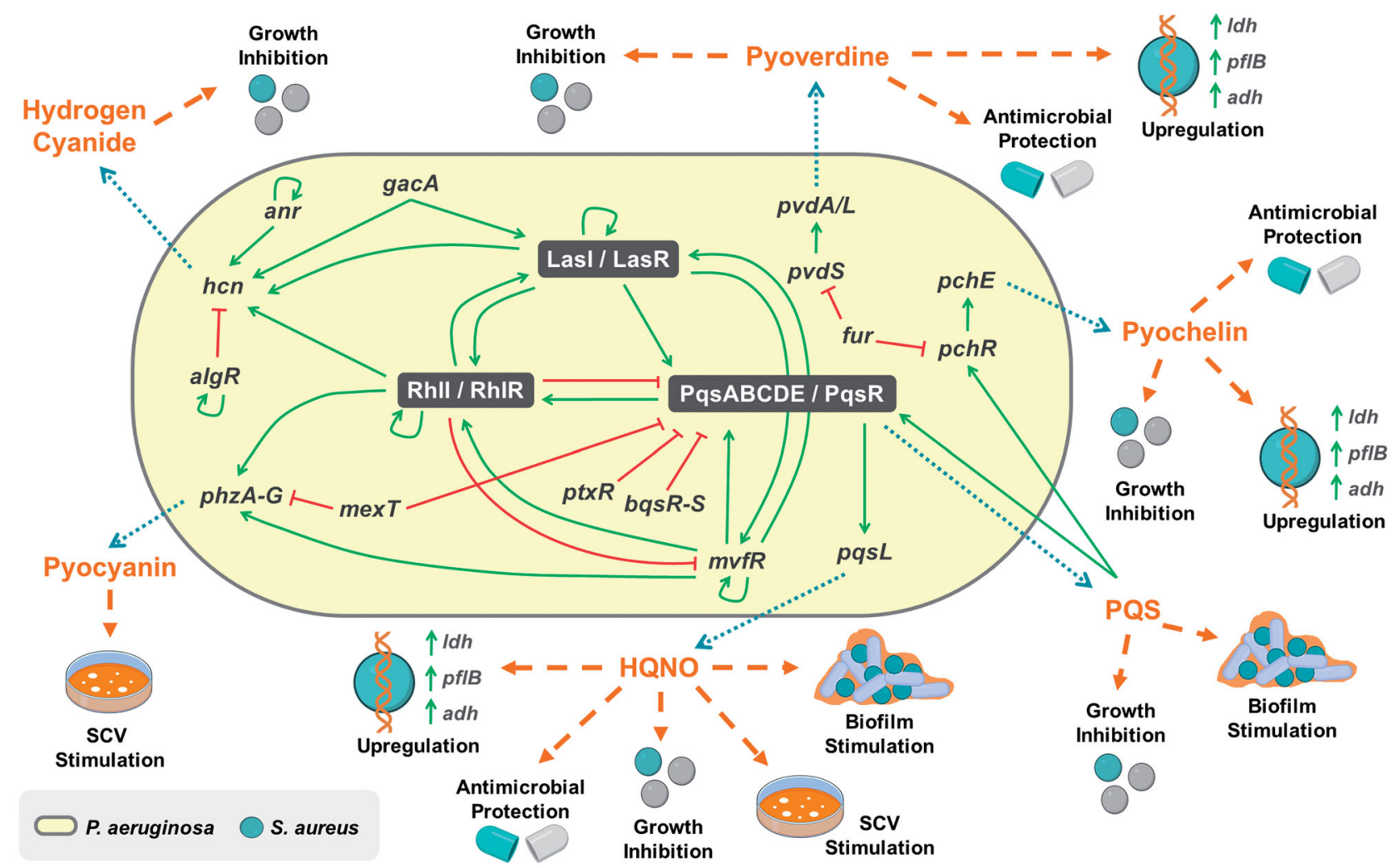

Figure 5. Schematic representation of the effect of $P$. aeruginosa on $S$. aureus in dual-species biofilms. Annotated interconnections between the molecules produced by $P$. aeruginosa, their effect on $S$. aureus, and the main regulatory network involved in $P$. aeruginosa $>$ S. aureus interactions is depicted. Solid arrows: upregulation of gene expression; lines with flat ends: downregulation of gene expression; dotted arrows: synthesis process; dashed arrows: effect on S. aureus.

(Bredenbruch et al. 2006). Specifically, PQS can prompt the expression of genes involved both in the regulation (pvdS) and biosynthesis of pyoverdine (e.g. pvdA and $p v d E$ ) and pyochelin (pchE) (Diggle et al. 2007). Despite the reported inhibitory effect of pyoverdine and pyochelin on S. aureus (Figure 5), both siderophores were found to be involved in its protection from antibiotic treatment in a biofilm model of CF infection (Orazi and O'Toole 2017).

In fact, the growth of $S$. aureus is not completely inhibited by $P$. aeruginosa. S. aureus has defence mechanisms that help it outcompete $P$. aeruginosa in the same infection, thus coexisting as a persister (Nair et al. 2014). For instance, $P$. aeruginosa excretes HQNO, which activates the alternative sigma factor $B$ in $S$. aureus and alters the expression of several virulence factors, including those that regulate adherence, invasiveness, and persistence within host cells, and facilitates the emergence of SCV (Mitchell et al. 2010). The SCV phenotype can survive in proximity with $P$. aeruginosa, which allows $S$. aureus persistence. The stimulation of biofilm formation by $\mathrm{PQS}$ and $\mathrm{HQNO}$ also contributes to this persistence, as biofilm-enclosed cells become more protected from external stresses (Fugère et al. 2014).

Although the exact regulatory mechanisms behind $P$. aeruginosa/S. aureus communication in biofilms are not fully clear, the reconstructed knowledge-networks highlight the complexity and multi-tiered regulatory processes by which $P$. aeruginosa controls expression of virulence factors that critically affect $S$. aureus, impacting the pathogenicity of the biofilm-related infection. Specifically, the networks revealed that $P$. aeruginosa QS signalling plays an important role in this dualspecies interplay, since three QS systems, namely the systems Lasl/LasR, Rhll/RhIR and PqsABCDE/PqsR, positively regulate the expression of specific $P$. aeruginosa virulence factors affecting $S$. aureus.

Besides QS, the integrated regulatory network of $P$. aeruginosa shows that other genes can also play key roles and control multiple pathways that trigger interspecies interactions. For example, PtxR is a transcriptional regulator that reduces the expression of the pqs $A B C D E$ operon (Figure 5), which negatively affects the production of pyocyanin (Carty et al. 2006). Stressful environmental conditions may also be controlling the differential expression of the regulatory cascade. For instance, $m v f R$ is involved in regulating critical physiological processes, being known to control the transcription of iron-related genes, under low-iron concentrations (Ochsner et al. 2002), and upregulating the transcription of the three QS systems and pyocyanin 
synthesis (Figure 5). Once intracellular iron levels are high, uptake systems and their regulators, including $m v f R$, are repressed by the fur transcriptional regulator (Troxell and Hassan 2013). This negative feedback loop also turns down the production of all other systems under mvfR control (e.g. pyocyanin and HQNO synthesis) (Maura et al. 2016). Under low oxygen or anoxia, anr controls hydrogen cyanide production and other cellular responses, including biofilm formation associated with chronic infections (Hammond et al. 2015).

\section{Targeting P. aeruginosa/S. aureus biofilm communication}

Antivirulence agents are presented as alternative therapeutics that can circumvent antibiotic resistance by targeting virulence factors rather than bacterial growth pathways (i.e. the target of traditional antibiotics) (Totsika 2016). This strategy is quite advantageous considering the large number of putative virulent targets (Allen et al. 2014). Since QS is the main virulence regulator in bacteria, inhibition of QS mechanisms using quorum quenching (QQ) agents appears to be a promising strategy to modulate the virulence of bacterial pathogens (Chan et al. 2015). Moreover, as the target may constitute an extracellular factor, the emergence and spread of resistance could be less likely (Fetzner 2015). Nevertheless, the selection of the target is of critical importance for the effectiveness of the antimicrobial strategy. In line with this, it is essential to understand the full dynamics of action of the targeted virulence factor as well as the dynamics of production (Dickey et al. 2017). The expression of certain virulence factors could be subjected to the control of several regulatory mechanisms other than the targeted QS system (Arya and Princy 2016).

Figure 6 summarizes the antivirulence agents that have so far shown inhibitory effect against two major virulence factors produced by $P$. aeruginosa and annotated as affecting $S$. aureus in biofilm settings, HQNO and siderophores (pyoverdine and pyochelin). A search was made in http://pcquorum.org/ for inhibitors targeting not only the molecules of interest but also their coding genes. A total of 25 and 19 reported agents were encountered inhibiting HQNO and siderophores, respectively, with four of them being active against both pyoverdine and pyochelin (Figure 6).

Although these antivirulence agents may be promising for attenuating $S$. aureus and $P$. aeruginosa resilience in co-infection scenarios, this antivirulence strategy has to face several challenges to become a feasible therapeutic option. One of the challenges in disrupting QS networks is the fact that, in some cases, the interference with the target could promote virulence instead of attenuating it (García-Contreras et al. 2015). This point is even more relevant when considering polymicrobial infections. Because inter-species interactions could be mediate by QS-controlled factors, the interference with a QS system in a pathogen potentially could facilitate the pathogenicity of the co-infecting species (Radlinski et al. 2017). For example, the PqsABCDE/PqsR system influences the production of extracellular DNA (Whitchurch et al. 2002) and lectins, which influences biofilm formation and enhances colonisation and infection establishment in $P$. aeruginosa (Lee and Zhang 2015). In addition, this system positively regulates HQNO levels, involved in $P$. aeruginosa/ S. aureus interactions (Figure 5). Depending on the environmental conditions found by these pathogens, different interactions were annotated, including stimulation of S. aureus SCV, antimicrobial protection, and inhibition of $S$. aureus growth. Therefore, the interference with the QS system could reduce the overall virulence, by inhibiting $P$. aeruginosa biofilm formation and possibly preventing the formation of $S$. aureus SCV. On the other hand, as HQNO inhibits $S$. aureus growth, the antivirulence therapies targeting this molecule could backfire and facilitate the spreading of $S$. aureus.

Consequently, the choice of antivirulence agents must pay attention to the multi-species community as a whole, that is, the impact on the remaining cooccurring pathogens must be considered as it can have a positive, hence undesired, effect on them. Moreover, to increase antimicrobial effectiveness in polymicrobial communities, empiric therapy would probably require the combination with other antimicrobial agents (including other antivirulence drugs or even antibiotics). Very few studies have tackled this approach in doublespecies biofilms of $P$. aeruginosa and $S$. aureus. The only example found in the literature using an adapted optimized query is the use of the QS inhibitor hamamelitannin targeting the RAP/TRAP QS system in $S$. aureus, which was combined with vancomycin in a gauze and successfully reduced biofilm formation of both species in an in vitro mixed biofilm model of chronic wound (Brackman et al. 2016). Although antivirulence approaches have substantially progressed, it appears that the search for antivirulence agents is still a challenging and unexplored area of investigation, with most of these antivirulence drugs tested in vitro and against single-species populations (Pérez-Pérez et al. 2017; Martínez et al. 2019).

Overall, this work was able to decipher the current knowledge on the complex interactions between $P$. 
(A)

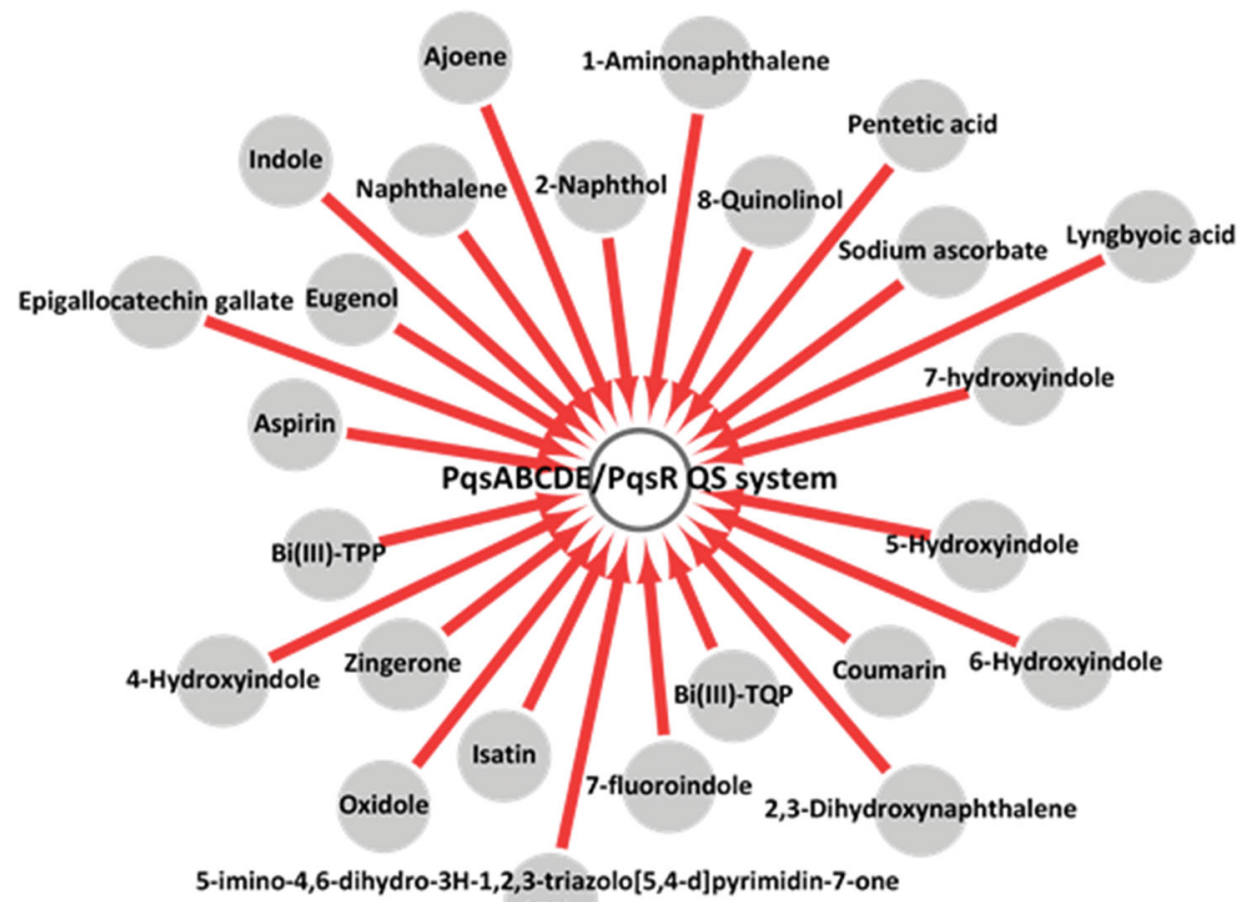

(G1)

(B)

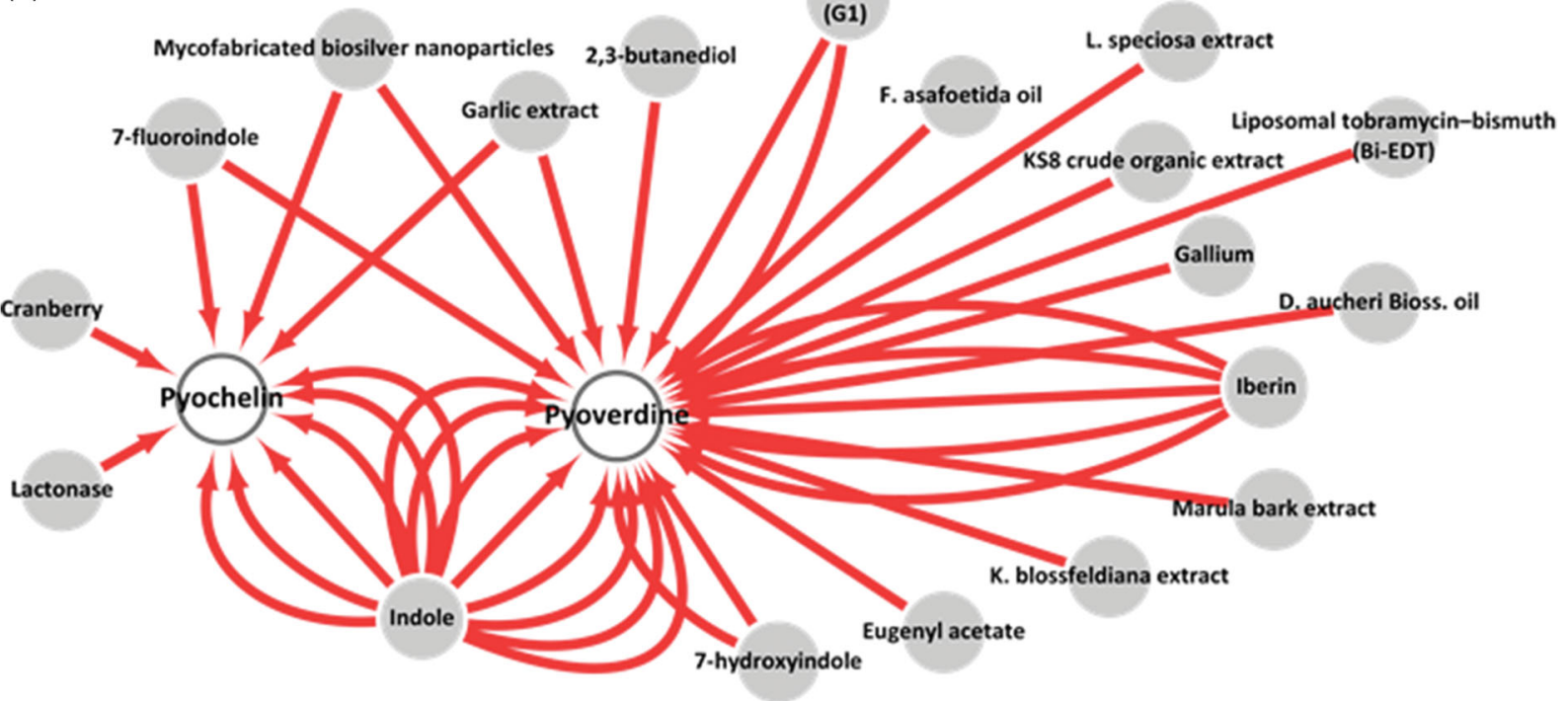

Figure 6. Networks of antivirulence agents targeting (A) the PqsABCDE/PqsR QS system and (B) the siderophores pyoverdine and pyochelin.

aeruginosa and $S$. aureus in the context of their competitiveness within the biofilm mode of growth, by implementing an innovative and systematic analysis of the scientific literature on this subject. The examination of the gathered information resulted in the clarification of the amount and types of studies being conducted, but more importantly, in the identification of the major and most studied molecular players in $P$. aeruginosa/S. aureus communication and/or interaction. More specifically, with $P$. aeruginosa revealed as key player, pseudomonal factors affecting $S$. aureus were pointed out (e.g. HQNO and siderophores) and their respective antivirulence agents identified. Yet, we are still far away from fully understanding the dynamic and complex networks of interactions that occur in the natural infection environment. Although efforts are being made to deeply understand the interactions that those microorganisms experience during infection, as this work shows, the percentage of studies that have pointed out specific mechanisms of interaction remains relatively 
small. The improvement of more powerful and efficient genetic tools and the design of feasible in vitro models that reflect the in vivo environment are pivotal for exploring unanswered questions about the ecology of $P$. aeruginosa/S. aureus biofilm infections. Expectantly, this work and its resulting database (www.ceb.uminho. pt/ISCTD.) will assist researchers aiming at diminishing the resilience of this bacterial consortium within biofilm related infections by downsizing time and work related costs.

\section{Disclosure statement}

No potential conflict of interest was reported by the authors.

\section{Funding}

This work was supported by the Portuguese Foundation for Science and Technology (FCT) under the scope of the strategic funding of UID/BIO/04469/2019 unit; the European Regional Development Fund under the scope of Norte2020 Programa Operacional Regional do Norte for the BioTecNorte operation (NORTE-01-0145-FEDER-000004); the COMPETE2020 and the FCT under the scope of the project POCI-01-0145FEDER-029841; and the FCT under the scope of the PhD Grant of Andreia Patricia Magalhães [grant number SFRH/BD/ 132165/2017].

\section{ORCID}

Andreia Patrícia Magalhães (D) http://orcid.org/0000-00032049-0185

Paula Jorge (D) http://orcid.org/0000-0002-0094-7830

Maria Olívia Pereira (iD http://orcid.org/0000-0002-4307-3985

\section{References}

Allen RC, Popat R, Diggle SP, Brown SP. 2014. Targeting virulence: can we make evolution-proof drugs? Nat Rev Microbiol. 12(4):300-308.

Allesen-Holm M, Barken KB, Yang L, Klausen M, Webb JS, Kjelleberg S, Molin S, Givskov M, Tolker-Nielsen T. 2006. A characterization of DNA release in Pseudomonas aeruginosa cultures and biofilms. Mol Microbiol. 59(4): 1114-1128.

Alves PM, Al-Badi E, Withycombe C, Jones PM, Purdy KJ, Maddocks SE. 2018. Interaction between Staphylococcus aureus and Pseudomonas aeruginosa is beneficial for colonisation and pathogenicity in a mixed biofilm. Pathog Dis. 76:fty003.

Archer NK, Mazaitis MJ, Costerton JW, Leid JG, Powers ME, Shirtliff ME. 2011. Staphylococcus aureus biofilms: properties, regulation, and roles in human disease. Virulence. 2(5):445-459.

Armbruster CR, Wolter DJ, Mishra M, Hayden HS, Radey MC, Merrihew G, MacCoss MJ, Burns J, Wozniak DJ, Parsek MR, et al. 2016. Staphylococcus aureus protein a mediates interspecies interactions at the cell surface of Pseudomonas aeruginosa. MBio. 7(3):e00538-16.

Arya R, Princy SA. 2016. Exploration of modulated genetic circuits governing virulence determinants in Staphylococcus aureus. Indian J Microbiol. 56(1):19-27.

Balasubramanian D, Schneper L, Kumari H, Mathee K. 2013. A dynamic and intricate regulatory network determines Pseudomonas aeruginosa virulence. Nucleic Acids Res. 41(1):1-20.

Bhattacharya M, Wozniak DJ, Stoodley P, Hall-Stoodley L. 2015. Prevention and treatment of Staphylococcus aureus biofilms. Expert Rev anti Infect Ther. 13(12):1499-1516.

Biswas L, Biswas R, Schlag M, Bertram R, Götz F. 2009. Smallcolony variant selection as a survival strategy for Staphylococcus aureus in the presence of Pseudomonas aeruginosa. Appl Environ Microbiol. 75(21):6910-6912.

Boucher HW, Talbot GH, Bradley JS, Edwards JE, Gilbert D, Rice LB, Scheld M, Spellberg B, Bartlett J. 2009. Bad bugs, no drugs: no ESKAPE! An update from the Infectious Diseases Society of America. Clin Infect Dis. 48(1):1-12.

Brackman G, Garcia-Fernandez MJ, Lenoir J, De Meyer L, Remon JP, De Beer T, Concheiro A, Alvarez-Lorenzo C, Coenye T. 2016. Dressings loaded with cyclodextrinhamamelitannin complexes increase Staphylococcus aureus susceptibility toward antibiotics both in single as well as in mixed biofilm communities. Macromol Biosci. 16(6): 859-869.

Brandel J, Humbert N, Elhabiri M, Schalk IJ, Mislin GLA, Albrecht-Gary A-M. 2012. Pyochelin, a siderophore of Pseudomonas aeruginosa: Physicochemical characterization of the iron(iii), copper(ii) and zinc(ii) complexes. Dalton Trans. 41(9):2820.

Bredenbruch F, Geffers R, Nimtz M, Buer J, Haussler S. 2006. The Pseudomonas aeruginosa quinolone signal (PQS) has an iron-chelating activity. Environ Microbiol. 8(8): 1318-1329.

Breidenstein EBM, de la Fuente-Núñez C, Hancock R. 2011. Pseudomonas aeruginosa: all roads lead to resistance. Trends Microbiol. 19(8):419-426.

Brown S, Cornforth D, Mideo N. 2012. Evolution of virulence in opportunistic pathogens: generalism, plasticity, and control. Trends Microbiol. 20(7):336-342.

Carty NL, Layland N, Colmer-Hamood JA, Calfee MW, Pesci EC, Hamood AN. 2006. PtxR modulates the expression of QS-controlled virulence factors in the Pseudomonas aeruginosa strain PAO1. Mol Microbiol. 61(3):782-794.

Castillo-Juárez I, Maeda T, Mandujano-Tinoco EA, Tomás M, Pérez-Eretza B, García-Contreras SJ, Wood TK, GarcíaContreras R. 2015. Role of quorum sensing in bacterial infections. WJCC. 3(7):575-598.

Chan KG, Liu YC, Chang CY. 2015. Inhibiting N-acylhomoserine lactone synthesis and quenching Pseudomonas quinolone quorum sensing to attenuate virulence. Front Microbiol. 6:1173.

Cheung GYC, Joo H-S, Chatterjee SS, Otto M. 2014. Phenolsoluble modulins - critical determinants of staphylococcal virulence. FEMS Microbiol Rev. 38(4):698-719.

Cheung GYC, Wang R, Khan BA, Sturdevant DE, Otto $M$. 2011. Role of the accessory gene regulator agr in community-associated methicillin-resistant Staphylococcus aureus pathogenesis. Infect Immun. 79(5):1927-1935. 
Chopra I, Schofield C, Everett M, O'Neill A, Miller K, Wilcox M, Frère J-M, Dawson M, Czaplewski L, Urleb U, et al. 2008. Treatment of health-care-associated infections caused by Gram-negative bacteria: a consensus statement. Lancet Infect Dis. 8(2):133-139.

Ciofu O, Tolker-Nielsen T, Jensen PØ, Wang H, Høiby N. 2015. Antimicrobial resistance, respiratory tract infections and role of biofilms in lung infections in cystic fibrosis patients. Adv Drug Deliv Rev. 85:7-23.

Ciulla M, Di Stefano A, Marinelli L, Cacciatore I, Di Biase G. 2019. RNAlll inhibiting peptide (RIP) and derivatives as potential tools for the treatment of $S$. aureus biofilm infections. CTMC. 18(24):2068-2079.

Costerton JW. 2001. Cystic fibrosis pathogenesis and the role of biofilms in persistent infection. Trends Microbiol. 9(2): $50-52$.

Davey M. E, Caiazza N. C, O'Toole G. A. 2003. Rhamnolipid surfactant production affects biofilm architecture in Pseudomonas aeruginosa PAO1. J Bacteriol. 185(3): 1027-1036.

DeLeon S, Clinton A, Fowler H, Everett J, Horswill AR, Rumbaugh KP. 2014. Synergistic interactions of Pseudomonas aeruginosa and Staphylococcus aureus in an in vitro wound model. Infect Immun. 82(11):4718-4728.

Dickey SW, Cheung GYC, Otto M. 2017. Different drugs for bad bugs: Antivirulence strategies in the age of antibiotic resistance. Nat Rev Drug Discov. 16(7):457-471.

Diggle SP, Matthijs S, Wright VJ, Fletcher MP, Chhabra SR, Lamont IL, Kong X, Hider RC, Cornelis P, Cámara M, et al. 2007. The Pseudomonas aeruginosa 4-quinolone signal molecules $\mathrm{HHQ}$ and $\mathrm{PQS}$ play multifunctional roles in quorum sensing and iron entrapment. Chem Biol. 14(1):87-96.

Dixon EF, Hall RA. 2015. Noisy neighbourhoods: quorum sensing in fungal-polymicrobial infections. Cell Microbiol. 17(10):1431-1441.

Dubern J-F, Diggle SP. 2008. Quorum sensing by 2-alkyl-4quinolones in Pseudomonas aeruginosa and other bacterial species. Mol Biosyst. 4(9):882-888.

Fetzner S. 2015. Quorum quenching enzymes. J Biotechnol. 201:2-14.

Fey PD. 2010. Modality of bacterial growth presents unique targets: how do we treat biofilm-mediated infections? Curr Opin Microbiol. 13(5):610-615.

Filkins L M, Graber J A, Olson D G, Dolben E L, Lynd L R, Bhuju S, O'Toole G A. 2015. Coculture of Staphylococcus aureus with Pseudomonas aeruginosa drives $S$. aureus towards fermentative metabolism and reduced viability in a cystic fibrosis model. J Bacteriol. 197(14):2252-2264.

Fisher EL, Otto M, Cheung G. 2018. Basis of virulence in enterotoxin-mediated staphylococcal food poisoning. Front Microbiol. 9:436.

Folkesson A, Jelsbak L, Yang L, Johansen HK, Ciofu O, Hoiby N, Molin SS, Høiby N, Molin SS. 2012. Adaptation of Pseudomonas aeruginosa to the cystic fibrosis airway: An evolutionary perspective. Nat Rev Microbiol. 10(12): 841-851.

Foster TJ, Geoghegan JA, Ganesh VK, Höök M. 2014. Adhesion, invasion and evasion: the many functions of the surface proteins of Staphylococcus aureus. Nat Rev Microbiol. 12(1):49-62.

Frimmersdorf E, Horatzek S, Pelnikevich A, Wiehlmann L, Schomburg D. 2010. How Pseudomonas aeruginosa adapts to various environments: a metabolomic approach. Environ Microbiol. 12(6):1734-1747.

Fugère A, Séguin DL, Mitchell G, Déziel E, Dekimpe V, Cantin AM, Frost E, Malouin F. 2014. Interspecific small molecule interactions between clinical isolates of Pseudomonas aeruginosa and Staphylococcus aureus from adult cystic fibrosis patients. PLoS One. 9(1):e86705.

Garcia LG, Lemaire S, Kahl BC, Becker K, Proctor RA, Denis O, Tulkens PM, Van Bambeke F. 2013. Antibiotic activity against small-colony variants of Staphylococcus aureus: review of in vitro, animal and clinical data. J Antimicrob Chemother. 68(7):1455-1464.

García-Contreras R, Peréz-Eretza $B$, Jasso-Chávez R, Lira-Silva E, Roldán-Sánchez J A, González-Valdez A, Soberón-Chávez G, Coria-Jiménez R, Martínez-Vázquez $M$, Alcaraz $L D$, et al. 2015. High variability in quorum quenching and growth inhibition by furanone C-30 in Pseudomonas aeruginosa clinical isolates from cystic fibrosis patients. Pathog Dis. 73(6):ftv040.

Goerke C, Campana S, Bayer MG, Döring G, Botzenhart K, Wolz C. 2000. Direct quantitative transcript analysis of the agr regulon of Staphylococcus aureus during human infection in comparison to the expression profile in vitro. Infect Immun. 68(3):1304-1311.

Grandclément $C$, Tannières $M$, Moréra S, Dessaux Y, Faure D. 2016. Quorum quenching: role in nature and applied developments. FEMS Microbiol Rev. 40(1):86-116.

Hall S, McDermott $C$, Anoopkumar-Dukie $S$, McFarland A, Forbes A, Perkins A, Davey A, Chess-Williams R, Kiefel M, Arora D, et al. 2016. Cellular effects of pyocyanin, a secreted virulence factor of Pseudomonas aeruginosa. Toxins. 8(8):236.

Hammond JH, Dolben EF, Smith TJ, Bhuju S, Hogan DA. 2015. Links between Anr and quorum sensing in Pseudomonas aeruginosa biofilms. J Bacteriol. 197(17): 2810-2820.

Hauser AR. 2009. The type III secretion system of Pseudomonas aeruginosa: infection by injection. Nat Rev Microbiol. 7(9):654-665.

Hawver LA, Jung SA, Ng WL. 2016. Specificity and complexity in bacterial quorum-sensing systems. FEMS Microbiol Rev. 40(5):738-752.

Hoffman L. R, Deziel E, D'Argenio D. A, Lepine F, Emerson J, McNamara S, Gibson R. L, Ramsey B. W, Miller S. I. 2006. Selection for Staphylococcus aureus small-colony variants due to growth in the presence of Pseudomonas aeruginosa. Proc Natl Acad Sci USA. 103(52):19890-19895.

Høiby N. 2011. Recent advances in the treatment of Pseudomonas aeruginosa infections in cystic fibrosis. BMC Med. 9(1):32.

Jakobsen TH, Bjarnsholt T, Jensen PØ, Givskov M, Høiby N. 2013. Targeting quorum sensing in Pseudomonas aeruginosa biofilms: current and emerging inhibitors. Future Microbiol. 8(7):901-921.

Jennings MT, Dasenbrook EC, Lechtzin N, Boyle MP, Merlo CA. 2017. Risk factors for persistent methicillin-resistant Staphylococcus aureus infection in cystic fibrosis. J Cyst Fibros. 16(6):681-686.

Jorge P, Lourenço A, Pereira MO. 2012. New trends in peptide-based anti-biofilm strategies: a review of recent achievements and bioinformatic approaches. Biofouling. 28(10):1033-1061. 
Jorge $P$, Magalhães AP, Grainha T, Alves D, Sousa AM, Lopes SP, Pereira MO. 2019. Antimicrobial resistance three ways: healthcare crisis, major concepts and the relevance of biofilms. FEMS Microbiol Ecol. 95.

Kazmierczak BI, Schniederberend M, Jain R. 2015. Crossregulation of Pseudomonas motility systems: the intimate relationship between flagella, pili and virulence. Curr Opin Microbiol. 28:78-82.

Knecht L D, O'Connor G, Mittal R, Liu X Z, Daftarian P, Deo S K, Daunert S. 2016. Serotonin activates bacterial quorum sensing and enhances the virulence of Pseudomonas aeruginosa in the host. EBioMedicine. 9:161-169.

Kong C, Neoh H, Nathan S. 2016. Targeting Staphylococcus aureus toxins: A potential form of anti-virulence therapy. Toxins. 8(3):72.

Le KY, Otto M. 2015. Quorum-sensing regulation in staphylococci-an overview. Front Microbiol. 6:1174.

Lee J, Wu J, Deng Y, Wang J, Wang C, Wang J, Chang C, Dong Y, Williams P, Zhang LH. 2013. A cell-cell communication signal integrates quorum sensing and stress response. Nat Chem Biol. 9(5):339-343.

Lee J, Zhang L. 2015. The hierarchy quorum sensing network in Pseudomonas aeruginosa. Protein Cell. 6(1):26-41.

Lequette Y, Greenberg EP. 2005. Timing and localization of rhamnolipid synthesis gene expression in Pseudomonas aeruginosa biofilms. J Bacteriol. 187(1):37-44.

Lister JL, Horswill AR. 2014. Staphylococcus aureus biofilms: Recent developments in biofilm dispersal. Front Cell Infect Microbiol. 4:178.

Liu Q, Yeo W, Bae T. 2016. The SaeRS two-component system of Staphylococcus aureus. Genes. 7(10):81.

Lyczak JB, Cannon CL, Pier GB. 2002. Lung infections associated with cystic fibrosis. Clin Microbiol Rev. 15(2):194-222.

Martínez OF, Cardoso MH, Ribeiro SM, Franco OL. 2019. Recent advances in anti-virulence therapeutic strategies with a focus on dismantling bacterial membrane microdomains, toxin neutralization, quorum-sensing interference and biofilm inhibition. Front Cell Infect Microbiol. 9:74.

Mashburn LM, Jett AM, Akins DR, Whiteley M. 2005. Staphylococcus aureus serves as an iron source for Pseudomonas aeruginosa during in vivo coculture. J Bacteriol. 187(2):554-566.

Maura D, Hazan R, Kitao T, Ballok AE, Rahme LG. 2016. Evidence for direct control of virulence and defense gene circuits by the Pseudomonas aeruginosa quorum sensing regulator, MvfR. Sci Rep. 6(1):1-14.

Michalska M, Wolf P. 2015. Pseudomonas exotoxin A: Optimized by evolution for effective killing. Front Microbiol. 6:963.

Miller CL, Van Laar TA, Chen T, Karna SLR, Chen P, You T, Leung KP. 2017. Global transcriptome responses including small RNAs during mixed-species interactions with methicillin-resistant Staphylococcus aureus and Pseudomonas aeruginosa. Microbiol Open. 6:e00427.

Mitchell G, Séguin DL, Asselin A-E, Déziel E, Cantin AM, Frost EH, Michaud S, Malouin F. 2010. Staphylococcus aureus sigma B-dependent emergence of small-colony variants and biofilm production following exposure to Pseudomonas aeruginosa 4-hydroxy-2-heptylquinoline-Noxide. BMC Microbiol. 10(1):33.

Mulcahy LR, Isabella VM, Lewis K. 2014. Pseudomonas aeruginosa biofilms in disease. Microb Ecol. 68(1):1-12.
Nair N, Biswas R, Götz F, Biswas L. 2014. Impact of Staphylococcus aureus on pathogenesis in polymicrobial infections. Infect Immun. 82(6):2162-2169.

Nguyen AT, Oglesby-Sherrouse AG. 2015. Spoils of war: iron at the crux of clinical and ecological fitness of Pseudomonas aeruginosa. Biometals. 28(3):433-443.

Ochsner UA, Wilderman PJ, Vasil Al, Vasil ML. 2002. GeneChip expression analysis of the iron starvation response in Pseudomonas aeruginosa: identification of novel pyoverdine biosynthesis genes. Mol Microbiol. 45(5): 1277-1287.

Orazi G, O'Toole GA. 2017. Pseudomonas aeruginosa alters Staphylococcus aureus sensitivity to vancomycin in a biofilm model of cystic fibrosis infection. MBio. 8(4): e00873-17.

Otto M. 2014. Staphylococcus aureus toxins. Curr Opin Microbiol. 17:32-37.

Pader V, James EH, Painter KL, Wigneshweraraj S, Edwards AM. 2014. The Agr quorum-sensing system regulates fibronectin binding but not hemolysis in the absence of $a$ functional electron transport chain. Infect Immun. 82(10): 4337-4347.

Palmer KL, Mashburn LM, Singh PK, Whiteley M. 2005. Cystic fibrosis sputum supports growth and cues key aspects of Pseudomonas aeruginosa physiology. J Bacteriol. 187(15): 5267-5277.

Pang Z, Raudonis R, Glick BR, Lin TJ, Cheng Z. 2019. Antibiotic resistance in Pseudomonas aeruginosa: Mechanisms and alternative therapeutic strategies. Biotechnol Adv. 37(1):177-192.

Pérez-Pérez $M$, Jorge $P$, Pérez Rodríguez $G$, Pereira $M O$, Lourenço A. 2017. Quorum sensing inhibition in Pseudomonas aeruginosa biofilms: new insights through network mining. Biofouling. 33(2):128-142.

Pesci EC, Pearson JP, Seed PC, Iglewski BH. 1997. Regulation of las and rhl quorum sensing in Pseudomonas aeruginosa. J Bacteriol. 179(10):3127-3132.

Pietrocola G, Nobile G, Rindi S, Speziale P. 2017. Staphylococcus aureus manipulates innate immunity through own and host-expressed proteases. Front Cell Infect Microbiol. 7:166.

Price KE, Hampton TH, Gifford AH, Dolben EL, Hogan DA, Morrison HG, Sogin ML, O'Toole GA. 2013. Unique microbial communities persist in individual cystic fibrosis patients throughout a clinical exacerbation. Microbiome. 1(1):27.

Proctor RA, Kriegeskorte A, Kahl BC, Becker K, Löffler B, Peters G. 2014. Staphylococcus aureus small colony variants (SCVs): A road map for the metabolic pathways involved in persistent infections. Front Cell Infect Microbiol. 4:99.

Radlinski L, Rowe SE, Kartchner LB, Maile R, Cairns BA, Vitko NP, Gode CJ, Lachiewicz AM, Wolfgang MC, Conlon BP. 2017. Pseudomonas aeruginosa exoproducts determine antibiotic efficacy against Staphylococcus aureus. PLoS Biol. 15(11):e2003981.

Razvi S, Quittell L, Sewall A, Quinton H, Marshall B, Saiman L. 2009. Respiratory microbiology of patients with cystic fibrosis in the United States, 1995 to 2005. Chest. 136(6): 1554-1560.

Recsei P, Kreiswirth B, O'Reilly M, Schlievert P, Gruss A, Novick R. P. 1986. Regulation of exoprotein gene 
expression in Staphylococcus aureus by agar. Mol Gen Genet. 202(1):58-61.

Reinhart AA, Oglesby-Sherrouse AG. 2016. Regulation of Pseudomonas aeruginosa virulence by distinct iron sources. Genes. 7(12):126.

Rodrigo-Troyano A, Sibila O. 2017. The respiratory threat posed by multidrug resistant Gram-negative bacteria. Respirology. 22(7):1288-1299.

Rogers GB, Hoffman LR, Whiteley M, Daniels T V, Carroll MP, Bruce KD. 2010. Revealing the dynamics of polymicrobial infections: implications for antibiotic therapy. Trends Microbiol. 18(8):357-364.

Sayers EW, Beck J, Brister JR, Bolton EE, Canese K, Comeau DC, Funk K, Ketter A, Kim S, Kimchi A. 2019. Database resources of the National Center for Biotechnology Information. Nucleic Acids Res. 47(D1):D23-D28.

Schmidtchen A, Wolff H, Hansson C. 2001. Differential proteinase expression by Pseudomonas aeruginosa derived from chronic leg ulcers. Acta Derm Venereol. 81(6): 406-409.

Scoffone VC, Trespidi G, Chiarelli LR, Barbieri G, Buroni S. 2019. Quorum sensing as antivirulence target in cystic fibrosis pathogens. Int J Mol Sci. 20:pii: E1838.

Shannon P, Markiel A, Ozier O, Baliga NS, Wang JT, Ramage D, Amin N, Schwikowski B, Ideker T. 2003. Cytoscape: a software environment for integrated models of biomolecular interaction networks. Genome Res. 13(11):2498-2504.

Sharma G, Rao S, Bansal A, Dang S, Gupta S, Gabrani R. 2014. Pseudomonas aeruginosa biofilm: potential therapeutic targets. Biologicals. 42(1):1-7.

Shen K, Sayeed S, Antalis P, Gladitz J, Ahmed A, Dice B, Janto B, Dopico R, Keefe R, Hayes J, et al. 2006. Extensive genomic plasticity in Pseudomonas aeruginosa revealed by identification and distribution studies of novel genes among clinical isolates. Infect Immun. 74(9):5272-5283.

Shrout JD, Chopp DL, Just CL, Hentzer M, Givskov M, Parsek MR. 2006. The impact of quorum sensing and swarming motility on Pseudomonas aeruginosa biofilm formation is nutritionally conditional. Mol Microbiol. 62(5):1264-1277.

Silby MW, Winstanley C, Godfrey SAC, Levy SB, Jackson RW. 2011. Pseudomonas genomes: diverse and adaptable. FEMS Microbiol Rev. 35(4):652-680.

Sobin L, Kawai K, Irace AL, Gergin O, Cunningham M, Sawicki GS, Adil EA. 2017. Microbiology of the upper and lower airways in pediatric cystic fibrosis patients. Otolaryngol Head Neck Surg. 157(2):302-308.

Solano C, Echeverz M, Lasa I. 2014. Biofilm dispersion and quorum sensing. Curr Opin Microbiol. 18:96-104.

Stacy A, McNally L, Darch SE, Brown SP, Whiteley M. 2016. The biogeography of polymicrobial infection. Nat Rev Microbiol. 14(2):93-105.

Tognon M, Köhler T, Luscher A, Van Delden C. 2019. Transcriptional profiling of Pseudomonas aeruginosa and Staphylococcus aureus during in vitro co-culture. BMC Genomics. 20(1):30.

Totsika M. 2016. Benefits and challenges of antivirulence antimicrobials at the dawn of the post-antibiotic era. DDL. 6(1):30-37.

Troxell B, Hassan HM. 2013. Transcriptional regulation by ferric uptake regulator (Fur) in pathogenic bacteria. Front Cell Infect Microbiol. 3:59.
Tuchscherr L, Heitmann V, Hussain M, Viemann D, Roth J, von Eiff C, Peters G, Becker K, Löffler B. 2010. Staphylococcus aureus small-colony variants are adapted phenotypes for intracellular persistence. J Infect Dis. 202(7):1031-1040.

Tuchscherr L, Medina E, Hussain M, Völker W, Heitmann V, Niemann S, Holzinger D, Roth J, Proctor R A, Becker K, et al. 2011. Staphylococcus aureus phenotype switching: an effective bacterial strategy to escape host immune response and establish a chronic infection. EMBO Mol Med. 3(3):129-141.

Turner KH, Everett J, Trivedi U, Rumbaugh KP, Whiteley M. 2014. Requirements for Pseudomonas aeruginosa acute burn and chronic surgical wound infection. PLoS Genet. 10(7):e1004518.

Ueda A, Wood TK. 2009. Connecting quorum sensing, c-diGMP, pel polysaccharide, and biofilm formation in Pseudomonas aeruginosa through tyrosine phosphatase TpbA (PA3885). PLoS Pathog. 5(6):e1000483.

Venkatesan N, Perumal G, Doble M. 2015. Bacterial resistance in biofilm-associated bacteria. Future Microbiol. 10(11): 1743-1750.

Vuong C, Kocianova S, Yao Y, Carmody AB, Otto M. 2004. Increased colonization of indwelling medical devices by quorum-sensing mutants of Staphylococcus epidermidis in vivo. J Infect Dis. 190(8):1498-1505.

Vuong C, Saenz HL, Götz F, Otto M. 2000. Impact of the agr quorum-sensing system on adherence to polystyrene in Staphylococcus aureus. J Infect Dis. 182(6):1688-1693.

Waters CM, Bassler BL. 2005. Quorum sensing: cell-to-cell communication in bacteria. Annu Rev Cell Dev Biol. 21(1): 319-346.

Watkins RR, Holubar M, David MZ. 2019. Antimicrobial resistance in methicillin-resistant Staphylococcus aureus to newer antimicrobial agents. Antimicrob Agents Chemother. 63(12).

Wendlandt S, Schwarz S, Silley P. 2013. Methicillin-resistant Staphylococcus aureus: A food-borne pathogen? Annu Rev Food Sci Technol. 4(1):117-139.

Whitchurch CB, Tolker-Nielsen T, Ragas PC, Mattick JS. 2002. Extracellular DNA required for bacterial biofilm formation. Science. 295(5559):1487.

WHO 2017a. Guidelines for the prevention and control of carbapenem-resistant Enterobacteriaceae, Acinetobacter baumannii and Pseudomonas aeruginosa in health care facilities; [accessed 2019 Dec 5]. https://www.who.int/infection-prevention/publications/guidelines-cre/en/.

WHO 2017b. Global priority list of antibiotic-resistant bacteria to guide research, discovery, and development of new antibiotics; [accessed 2019 Dec 05]. https://www.who.int/ medicines/publications/global-priority-list-antibiotic-resistant-bacteria/en/.

Williams P, Cámara M. 2009. Quorum sensing and environmental adaptation in Pseudomonas aeruginosa: a tale of regulatory networks and multifunctional signal molecules. Curr Opin Microbiol. 12(2):182-191.

Woods PW, Haynes ZM, Mina EG, Marques C. 2018. Maintenance of $S$. aureus in co-culture with $P$. aeruginosa while growing as biofilms. Front Microbiol. 9:3291.

Zielinska AK, Beenken KE, Mrak LN, Spencer HJ, Post GR, Skinner RA, Tackett AJ, Horswill AR, Smeltzer MS. 2012. SarA-mediated repression of protease production plays a key role in the pathogenesis of Staphylococcus aureus USA300 isolates. Mol Microbiol. 86(5):1183-1196. 